Proc. Indian Âcad. Sci. (Earth Planet. Sci.), Vol. 95, No. 1, March 1986, pp. 1-12.

(C) Printed in India.

\title{
Power spectrum analysis of geomagnetic indices
}

\author{
R P KANE \\ Instituto de Pesquisas Espaciais-INPE, Conselho Nacional de Desenvolvimento Científico e \\ Tecnológico-CNPq, 12200 Sào José dos Campos, SP, Brasil \\ MS received 18 December 1984; revised 15 October 1985

\begin{abstract}
Using the method of maximum entropy spectral analysis (MESA) by Burg and the least-squares linear prediction (LSLP) (also called FABNE) by Barrodale and Erickson, the spectra of geomagnetic indices Ap, AN, AS, AE, AU, AL, Dst and cosmic ray neutron intensity at Deep River are obtained for 1965 and 1969 from daily means and for longer periods from monthly means. A large number of peaks of periodicities from 2 days to several years is obtained. many of which are shown by all the indices. Some of these are probably harmonics of the 27 days solar rotation period and the 20-22 years double sunspot cycle period. Comparison is made with results of earlier workers who reported fewer peaks.
\end{abstract}

Keywords. Power spectrum analysis; geomagnetic indices; maximum entropy spectral analysis; least-squares linear prediction; periodicity peaks.

\section{Introduction}

For detecting the periodicities in a given time series, the most popular method adopted till recently was that of Blackman and Tukey (1958). It is based on the autocorrelation function and one has to choose a lag factor $m$ and the power is finally obtained for periods $2 m, 2 m / 2,2 m / 3$ etc. Recently Burg (1967) proposed the method of maximum entropy spectral analysis (MESA) which gives a very good resolution in frequency even when the periods are almost comparable to the data length (see review by Ulrych and Bishop 1975). In the MESA, one chooses the length of the prediction error filter (LPEF). For low LPEF of the order of a few percent of the data length, only higher harmonics (smaller periodicities) start getting resolved. As LPEF is increased, larger and larger periodicities are revealed.

In spite of the superior resolution of the Burg method (Burg 1972), there are some serious drawbacks of this method viz, (i) a shift in the spectral lines, (ii) the occurrence of multiples, (iii) the difficulty in knowing the variance of the spectral density estimator and (iv) choice of the optimal length of the prediction error filter (LPEF). The problem of the proper choice of LPEF is the most tricky one. At very low values of LPEF, no worth while resolution is obtained. As LPEF is increased, more and more peaks in the low frequency side are revealed but the spectra become unstable, with the "explosion" of many peaks into multiples (Chen and Stegan 1974). For determining the optimum LPEF, Ulrych and Bishop (1975) suggested the use of the Akaike's (1969) final prediction error (FPE) criterion, and, if this failed (as happens often), an LPEF of about $50 \%$ of the data length was suggested to be generally adequate. Our own studies (Kane 1977, 1979) indicate that for samples containing peaks in a wide range of frequency, an LPEF of about $50 \%$ of data length was adequate to resolve frequencies exceeding the fifth harmonic, while for lower harmonics, LPEF even as high as $90 \%$ was sometimes needed, 
with the danger of peak-splitting and frequency shifts (specially in low frequencies) ever present.

The limitations of the Burg algorithm are caused by its imposition of a Toeplitz structure on the matrix of the system of equations which yield the AR (auto regressive) parameters. This procedure is responsible for the computational efficiency of the Burgs algorithm; but there is enough empirical evidence to show that the resulting spectra so obtained are inferior (in accuracy of frequency determination) to those obtained by using least-square (LS) solutions to the AR model (Ulrych and Clayton 1976).

Estimation of AR parameters by Ls methods has previously been unpopular because of the large computational effort involved, besides other reasons. However, Barrodale and Erickson $(1980 \mathrm{a}$, b) have recently developed an algorithm for solving the leastsquares linear prediction (LSLP) problem directly (without forcing a Toeplitz structure on the AR model). This algorithm, termed by them as FABNE, is claimed to be computationally efficient and numerically stable. Kane and Trivedi (1982) compared the Burg and FABNE spectra for some artificial samples and confirmed the superiority of FABNE over Burg spectra. Recently, Marple (1980) has also reported a new algorithm which is similar to that of Barrodale and Erickson except for some numerical sophistication and swiftness in calculation. In the present communication, we study the spectra of geomagnetic indices, by both the Burg algorithm (as given by Anderson 1974) and the FABNE method, mainly to locate the various periodicities $T_{n}$ (giving more weightage to the FABNE), using $\mathrm{LPEF}=50 \%$ or less for periodicities $T_{n}<T / 5$ and using LPEF exceeding $50 \%$ for periodicities $T_{n}>T / 5$ where $T=$ data length. Having picked up the possible $T_{n}$, we obtain the best estimates of the amplitudes $r_{n}$ and their standard errors $\sigma_{r n}$ using the expression

$$
\begin{aligned}
f(t) & =\sum^{n}\left(a_{n} \sin 2 \pi\left(t / T_{n}\right)+b_{n} \cos 2 \pi\left(t / T_{n}\right)\right) \\
& =\sum^{n} r_{n} \sin \left(2 \pi\left(t / T_{n}\right)+\phi_{n}\right),
\end{aligned}
$$

where $f(t)$ is the observed series, expressed as deviation from mean, and $t$ runs from 1 to data length $T$, and adopting standard statistical procedures involving a least-square fit (Johnston 1960; Bevington 1969). Amplitudes $r_{n}$ larger than $2 \sigma_{r n}$ are supposed to be significant at a $95 \%$ level.

Geomagnetic disturbances are graded by various indices viz, mid-latitude indices $\mathrm{Kp}$, Ap, AN, AS (Mayaud 1968), Dst (Sugiura and Poros 1971) and auroral region indices AE, AU, AL. Periodicities of 11 and 22 years have been reported (Cheronsky 1966; Russell and McPherron 1973). Mayaud (1970a, b) reported annual, semi-annual and diurnal variations. Shapley (1947) reported a 27-day recurrence tendency. Fraser-Smith (1972) obtained very prominent peaks at $35.6,16 \cdot 1,10 \cdot 2$ and 0.5 years, prominent peaks at $7 \cdot 04,5 \cdot 14,4 \cdot 10,1 \cdot 47,1.09$ years and $27 \cdot 2,27 \cdot 6$ days, moderate peaks at $54 \cdot 0,30 \cdot 5,26 \cdot 9$, $18 \cdot 7,14 \cdot 1,13 \cdot 7,13.6$ and $9 \cdot 39$ days and very weak peaks for 2-9 days, from a fast fourier transform analysis of 38 years data of Ap. Abdel-Wahab and Goned (1974) reported periods of 27 and 13.5 days. Burch (1973) showed that the auroral AU and AL indices had a seasonal as well as sector structure effect. It would be interesting to see what Burg and FABNE spectra for these parameters reveal. Currie (1976) had applied MESA to 100 annual values of the $a a$ magnetic activity index of Mayaud, using an $L P E F=30$, after removing the mean and linear trends. Courtillot et al (1977) repeated the same analysis 
with and without trend removals and showed (besides other features) that the removal of trend affected longer periods considerably. We now present our results.

\section{Data analysis}

Geomagnetic indices are known to have year-to-year variations. Hence, for the study of periodicities of a few days, it may be desirable to remove the long-term trends by using proper filters. We used daily mean values for the various parameters and obtained Burg and FABNE spectra from the original values as also after applying a high-pass (cutoff at 20 days) 51 point sine-termination filter (Behannon and Ness 1966). The results shown by both were almost similar except that peaks for periods exceeding about 20 were missing in the filtered data, as expected. Recently, Courtillot et al (1977) have also shown that Burg spectra of say a 100-year series with and without removing a linear or parabolic trend are the same for low periodicities (about 7 years or less) only. Hence, only analysis of unfiltered data is reported in what follows. Burg and FABNE spectra using 365 daily mean values for years of low and high solar activity (1965 and 1969 respectively) were obtained for the geomagnetic indices Dst, Ap, AN, AS, AE, AU, AL as also for the cosmic ray (CR) neutron monitor intensity at Deep River, Canada. Figure 1 shows the sample plot for the AN index for 1965 . For LPEF $=50$, very few peaks are revealed. For LPEF $=100$, many more peaks are revealed; but the number of peaks is

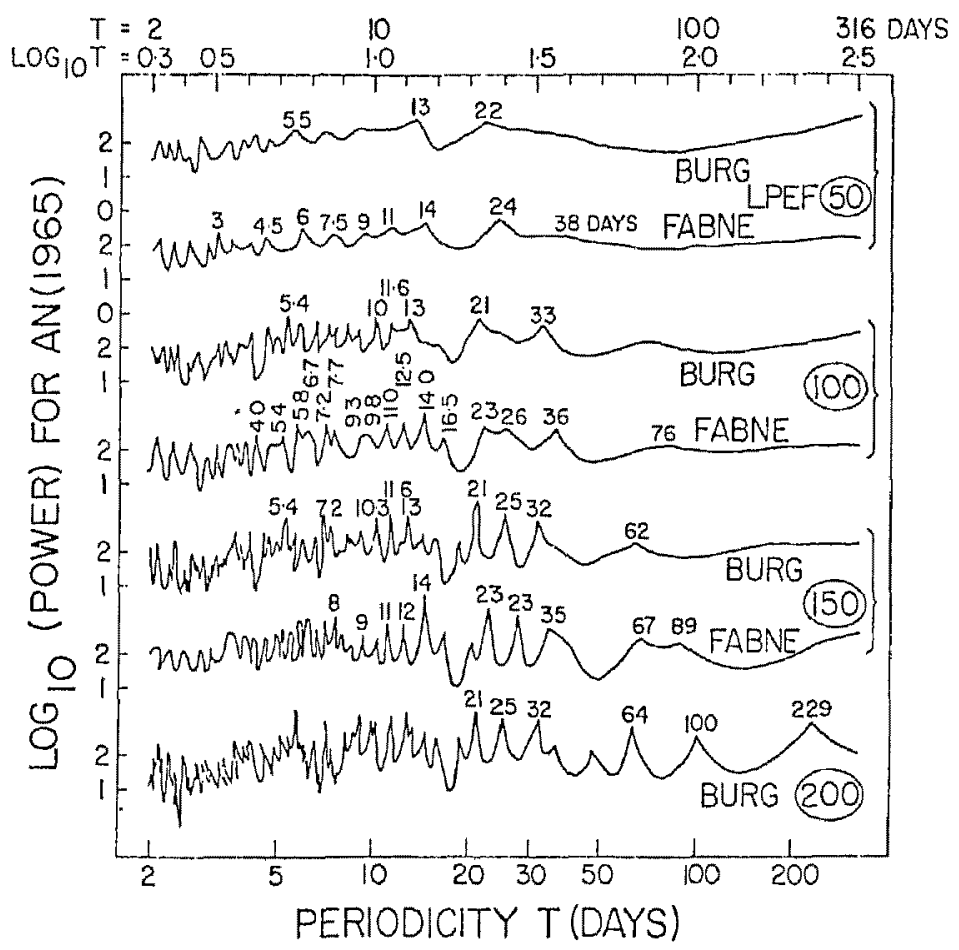

Figure 1. Burg and FABNE spectra for AN index for 365 days of 1965 for $L P E F=50,100,150$. For $L P E F=200$, only Burg spectra are shown as the FABNE program failed. 
lesser in the FabNe spectra. Probably there is some peak splitting in the Burg spectra. For $L P E F=150$, longer periodicities are revealed; but shorter periodicities show peak splitting in both Burg and FABNE spectra. Hence, for periodicities lesser than $T=20$ days, peaks were chosen from $F A B N E$ spectra for $L P E F=100$. For periodicities exceeding $T=20$ days, peaks were chosen from FABNE spectra for $L P E F=100$ and 150 . For $L P E F$ $=200$ or more the FABNE program failed. The Burg spectra for $\mathrm{LPEF}=200$ are shown at the bottom in figure 1, only for illustration. These were not used for choosing peaks.

Having chosen the peaks $T_{n}$ as mentioned above, these were used in (1) for estimating the amplitudes $r_{n}$ and their standard errors $\sigma_{r n}$. Periodicities (in days) for which $r_{n}$ exceeded $2 \sigma_{r n}$ are listed in table 1, for 1965 in the left half and for 1969 in the right half. In the middle column, the results of Fraser-Smith (1972) are also given for comparison. As can be seen, all the peaks seen by Fraser-Smith are revealed in our analysis also. In addition, our analysis reveals many more peaks. Thus, in 1965, significant peaks common to four or more indices were at about $4 \cdot 0,5 \cdot 4,5 \cdot 8,6 \cdot 7,7 \cdot 2,7 \cdot 6,9 \cdot 3,11 \cdot 0,12 \cdot 5$, $14 \cdot 0,16 \cdot 5,23,26,36,50$ and 66 days. In 1969 , the common peaks were at about $4 \cdot 0,9 \cdot 9$, $11 \cdot 0,12 \cdot 5,13 \cdot 5,14,17,20,29,35,48$ and 60 days. Peaks near 27 days are probably related to the solar rotation period and higher harmonics of the same at about $13 \cdot 5,9 \cdot 0,6 \cdot 7$, 5.5 days etc. seem to exist, as indicated in the last column of table 1. Other peaks could be harmonics of the 90 days ( 3 months) and 180 days ( 6 months) periods. The double solar rotation peak at 54 days reported by Fraser-Smith is not seen clearly in our analysis. Peaks near 7-8 days are indicated for many parameters. These peaks may be related to the sector structure of the interplanetary magnetic field (Burch 1973). AN and AS represent disturbance indices for the northern and southern hemisphere. For these, most of the peaks seem to be common in 1965. (For 1969, data for AN and AS were not available). For the auroral indices $\mathrm{AE}, \mathrm{AL}, \mathrm{AU}$, most of the peaks were common but not all, indicating a possibility of different physical mechanisms for AL and AU (Burch 1973). Whereas the significance of all these individual peaks cannot be guaranteed, their occurrence in more than one (often 3 or more) parameters leads us to believe that these peaks are genuine.

Table 1 and figure 1 indicate peaks in the long periodicity region ( $T=100$ days or more). However, in a 365 day data length, such long periodicities would be somewhat unreliable. These could be studied better by spectral analysis of monthly means. Such an analysis gave significant periodicities (in months). which are listed in table 2. The peaks observed by Fraser-Smith (1972) for 38 years of Ap data (1932-1970) by the fast fourier transform (FFT) method are given in the last column of table 2. Figure 2 shows the locations of these spectral peaks in the periodicity range 2-120 months. For Ap (full dots) and Dst, data used are monthly means for about 14 years (1957-70), for cosmic rays data are for 16 years (1958-73), for AL, AU, AE, data are for about 6 years (1965-70) and for AN, AS, data are for 4 years (1964-67). Periodicities are seen near 12 months and 6 months, in agreement with the annual and semi-annual waves reported by earlier workers (Mayaud 1970a; Fraser-Smith 1973) and also at 3 months. However, there seem to be many other peaks also common to many parameters. There is a prominent peak at about 15 months. Prominent peaks seem to occur at about 27 months also, similar to the quasibiennial oscillation ( $\mathrm{QBO}$ ) in meteorological parameters (Angell and Korshover 1964) and in the $H$ component of the geomagnetic field (Yacob and Bhargava 1968). Yet another prominent peak is at about 48 months (4 years). For Ap, we had data for a longer period also (1943-1970, 28 years). Spectral analysis gave significant peaks as indicated by crosses in figure 2 (just above the dots for Ap). Above 


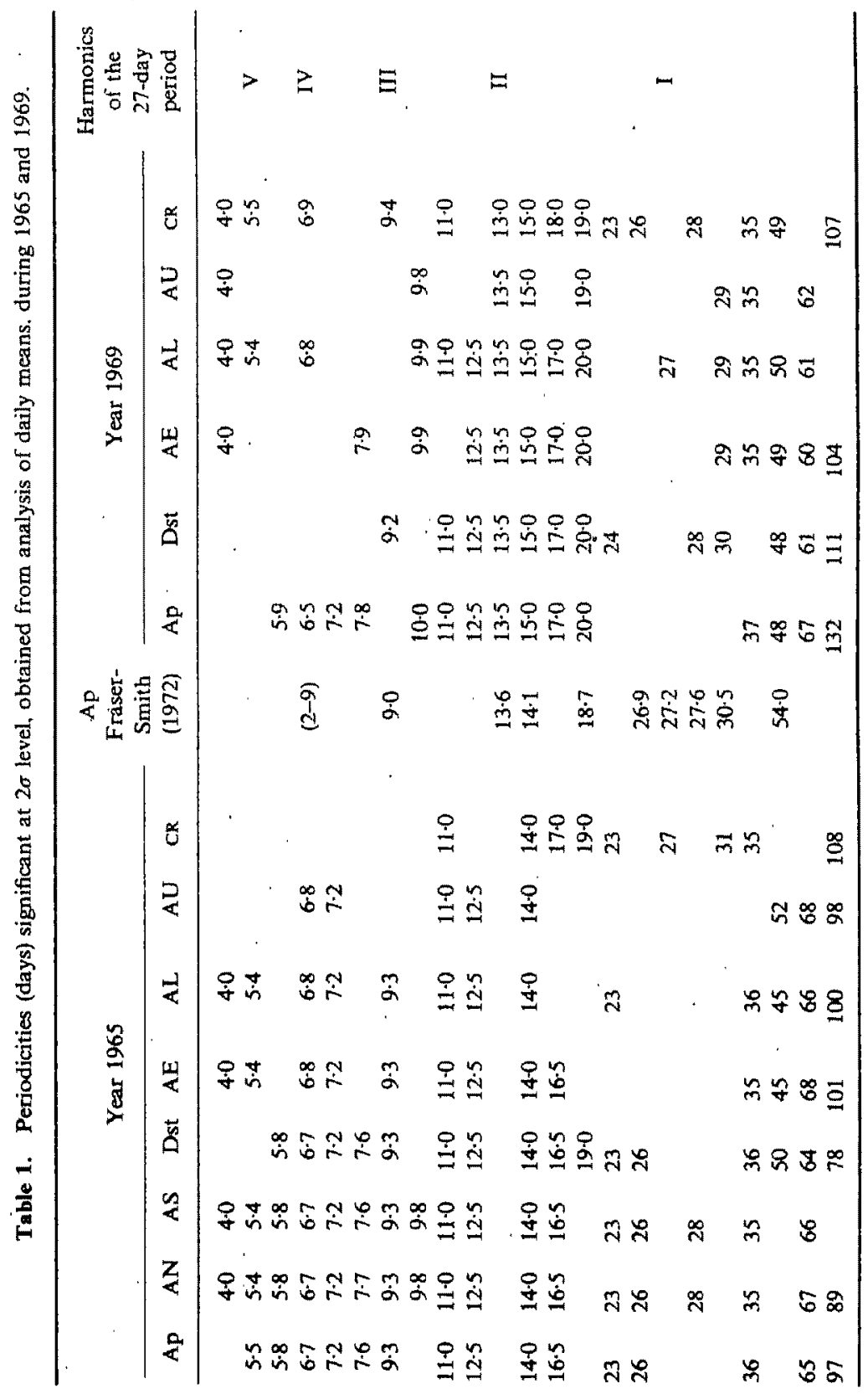




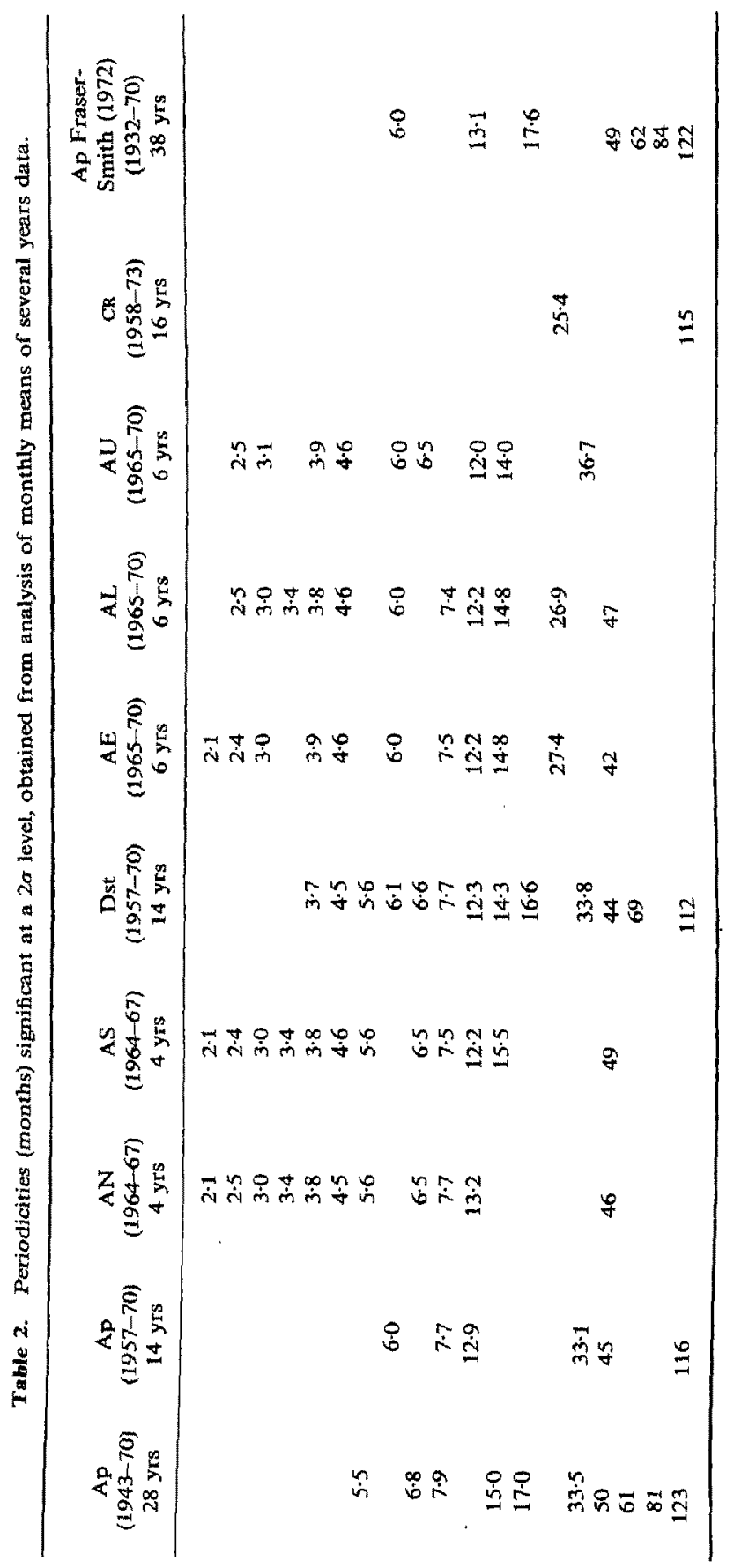




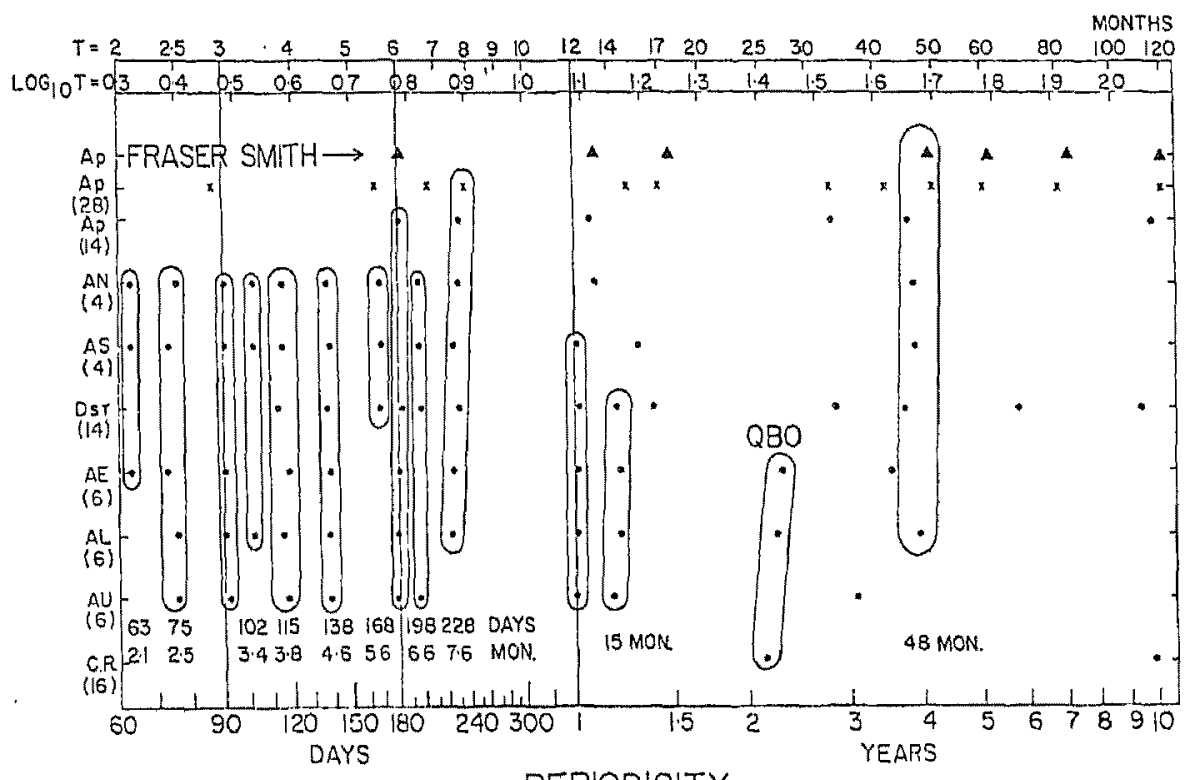

PERIODICITY

Figure 2. Locations of significant peaks of FABNE spectra (period $T=2-100$ months) of monthly means of various geomagnetic indices from data for 14 years (dots) and 28 years (crosses) for Ap, 14 years for Dst, 16 years for cosmic rays (CR), 6 years for AE, AU, AL, and 4 years for AS, AN. The triangles represent Ap peaks obtained by Fraser-Smith (1972) from 38 years data.

the crosses, the triangles show the peaks observed by Fraser-Smith (1972) for Ap for 38 years data. There are more crosses than triangles. Thus, our method reveals more peaks. The Ap peaks at 4.2, 5.1,6.8, 10.2 years look like harmonics of a 20-22 year double sunspot cycle. Currie $(1973,1974)$ obtained the Burg spectra of $H$ and $Z$ components of the geomagnetic field recorded at several observatories and reported several harmonics of the 10.5 and 21.4 year sunspot and double sunspot cycles and the annual wave. Courtillot and Le Mouël (1976) made a similar study and obtained some results slightly different from those of Currie.

Near 27 days, Fraser-Smith has reported two peaks at $T=27 \cdot 2$ and $27 \cdot 6$. To resolve this pair, the data length needed by the $1 /\left(f_{1}-f_{2}\right)$ criterion is about 1700 data points of daily means. Thus, results for daily means of any particular year as shown in table 1 or those for monthly mean for any number of years as shown in table 2 are not likely to resolve this pair. Hence, a separate analysis was carried out using about 7 years data of continuous daily means. For Ap, data for 28 years were divided into four groups of 7 years each as 1943-49,1950-56, 1957-63 and 1964-70. For Dst, 7 years groups were 1957-63, 1964-70. For AE, AU, AI and CR, only one group 1964-70 was made. Thus, in each of these groups the data length was about 2500 data points (daily means). Analysis of such large data lengths needs large computer times and hence the choice of LPEF was important. Since the period of 27 days is a very small fraction of 2500 days, we are dealing here with a very high harmonic (very low $T$ ) and hence low LPEF should be adequate. In figure 3 , we show the spectra for a limited range 2-40 days which includes the crucial period 27 days (marked by a vertical line) near which the peaks 27.2 and 27.6 


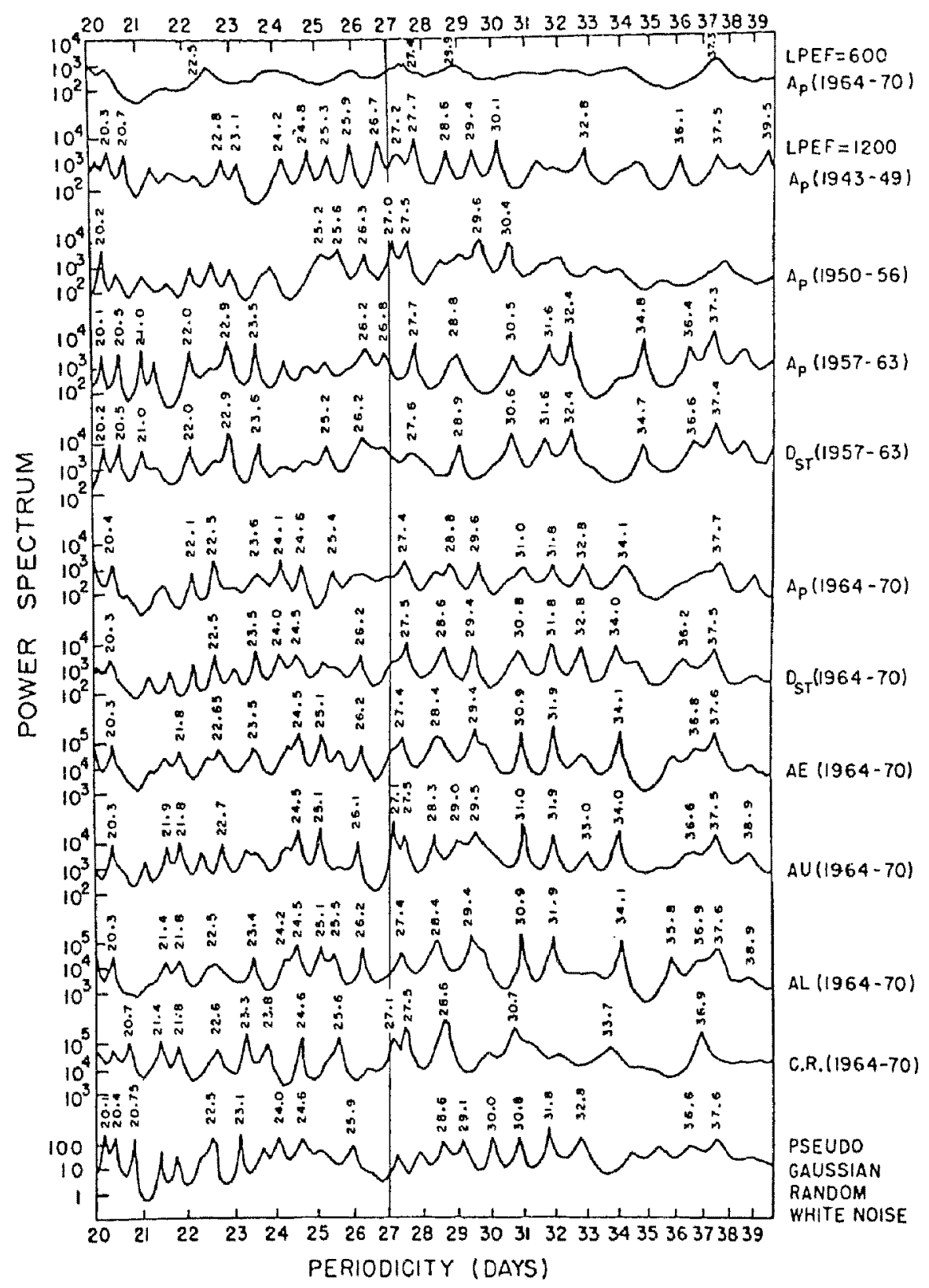

Figure 3. Spectra from about 2500 consecutive daily mean values of the various geomagnetic indices for LPEF of about $50 \%$ of total data length, for the restricted periodicity range $T$ $=20-40$ days (which includes the 27-day periodicity band) for several 7-year intervals as indicated. The top curve is for LPEF $=25 \%$ only for $A_{p}(1964-70)$. The bottom curve is for $\mathrm{LPEF}=50 \%$ for a 2500 data point sample of pseudo-Gaussian random noise.

days are expected. The top plot shows spectra for Ap for the 7 year period 1964-70 for an $\mathrm{LPEF}=600$, i.e., about $25 \%$ of the total data length. Only some broad peaks are revealed and we concluded that $25 \% \mathrm{LPEF}$ was inadequate. Hence, we chose LPEF $=1200$, i.e., about half data length for all further analysis. The resulting spectra are shown in 
figure 3, each plot representing a 7-year period. Even in the restricted range of 20-40 days, several peaks are noticed. Near 27 days, Ap shows peaks at 27.2 and 27.7, 27.0 and $27.5,26.8$ and 27.7 and 27.4 days for the four successive 7 -year periods. This could be because of a solar cycle control of the main solar rotation period of 27-37 days as suggested by Fraser-Smith (1973). In all other parameters, a prominent peak occurs at $27.55+0.15$ but only a small peak at $T=27 \cdot 1 \pm 0 \cdot 1$ is seen for some parameters. Thus, a major peak at 27.5 and a minor peak at 27.1 is indicated. However, equally (or sometimes more) prominent peaks are noticed at other values of $T$. The question of reliability of these again becomes important. We prepared a 2500 data point sample of pseudo-Gaussian random white noise and the spectra for the same for $L P E F=1200$ are shown as the bottom plot of figure 3. Many peaks are observed here too! Jensen and Ulrych (1973) calculated the probability distribution of the power density of such peaks. We noticed that in this sample of pseudo-Gaussian random noise for which the standard deviation chosen was 5 (variance 25), all peaks had a power less than about 140. For the 4 seven-yearly samples of Ap which we used, the standard deviations were $18,16,21$ and 12 and thus their variances were $13,10,18$ and 6 times the variance of the random samples. Thus, power densities up to about 1800,1400, 2300 and 900 for 1943-49, 1950-56, 1957-63 and 1964-70 respectively for Ap could be considered as suspect. Peaks having power exceeding these limits could be considered as genuine. Using these criteria, the peaks for which periodicities are labelled in figure 3 could be considered reliable. As can be seen, even these are many in number. In particular, peaks at about $T=20 \cdot 7 \pm 0 \cdot 3,22 \cdot 8 \pm 0 \cdot 3,24 \cdot 8 \pm 0 \cdot 3,25 \cdot 9 \pm 0 \cdot 3,27 \cdot 0 \pm 0 \cdot 2,27 \cdot 55 \pm 0 \cdot 15,28 \cdot 7$ $\pm 0 \cdot 3,29 \cdot 5 \pm 0 \cdot 1,30 \cdot 7 \pm 0 \cdot 3,31 \cdot 7 \pm 0 \cdot 2,32 \cdot 9 \pm 0 \cdot 1,34 \cdot 0 \pm 0 \cdot 1,34 \cdot 7 \pm 0 \cdot 1,36 \cdot 5 \pm 0 \cdot 3$, and $37.5 \pm 0.2$ days are observed prominently in many parameters.

It is tempting to identify $T=29.5$ days with the lunar synodic period. It must be noted, however, that some of these peaks tally rather well with the values observed in the random sample. Hence, there is some doubt about their genuineness.

In principle, one could do an analysis for 28 years data of continuous daily means. However, for a computer this would be a colossal task. As a naive alternative, we obtained an average plot for Ap by superposing the four plots for Ap in figure 3. The average showed peaks in bands as indicated above with a broad plateau in the region 26.6-27.8 days with a small peak only at about 27.6 days. We also tried using 7-day i.e. weekly means of Ap. From the 52 values per year, we obtained spectra for Ap for 1965 , $1966,1967,1968$ and 1969 . The significant periodicities are shown in figure 4 . There is a slight indication of a systematic shift of some periodicities from smaller to larger values from quiet sun to disturbed sun years. However, this could be a highly subjective judgement.

\section{Summary and conclusions}

The results of the present analysis may be summarized as follows:

(1) Burg and FABNE spectra of geomagnetic disturbance indices Ap, AN, AS, Dst, AE, $A U, A L$ and cosmic ray intensity CR were obtained for the quiet and disturbed solar activity years 1965 and 1969 respectively. Several significant periodicities (given in table 1) common to many parameters were noticed though these were not exactly identical for 1965 and 1969. In particular, whereas a $26-27$ day peak was prominent in 


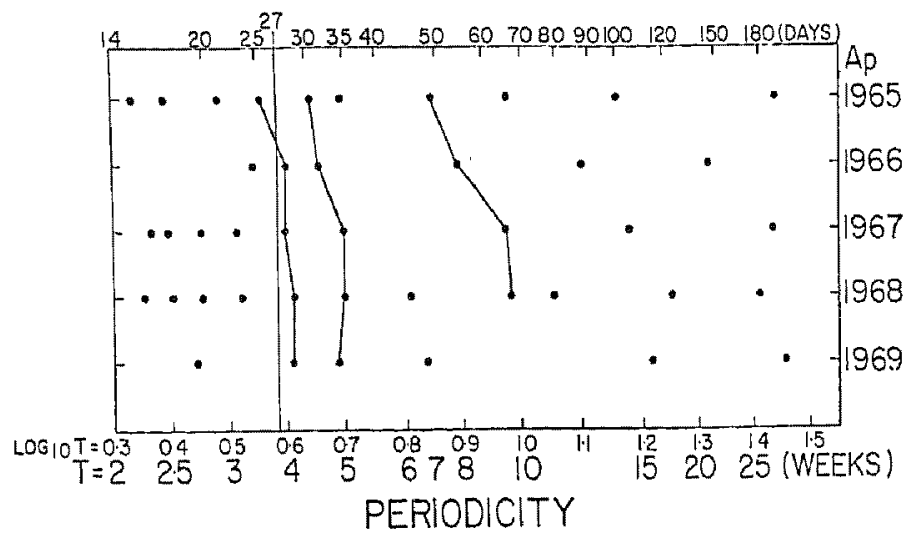

Figure 4. Spectral peaks (significant at a $2 \sigma$ level), from 52 weekly mean values of Ap for 1965, 1966, 1967, 1968 and 1969.

1965 , it was obscure in 1969 when a $28-29$ day periodicity was more prominent. This might be related to the observation of Svalgaard and Wilcox (1975) that whereas during most of the solar cycle a four-sector structure of interplanetary magnetic field is seen with a recurrence period of 27 days, near sunspot maximum, a superposed structure with polarity towards sun and a recurrence period of 28-29 days is observed. Periodicities could be identified as harmonics of the 27-day solar rotation period and possibly of the 90-day, 180-day and 360-day waves. Periodicities near about 8 days or less could be related to the sector structure of the interplanetary magnetic field. Occurrence of the same periodicities in cosmic ray intensity perhaps indicates modulation due to geomagnetic effects or a common cause in interplanetary space.

(2) Whereas AN and AS representing mid-latitude disturbances in northern and southern hemisphere show almost similar periodicities, AU and AL in the auroral zone show some differences indicating a partly different origin for the two (Burch 1973). (3) Analysis of about 7 years data of continuous daily means revealed several peaks in the $20-40$ days periodicity interval at about $20 \cdot 7,22 \cdot 8,24 \cdot 8,25 \cdot 9,27 \cdot 0,27 \cdot 6,28 \cdot 7,29 \cdot 5$, $30 \cdot 7,31 \cdot 7,32 \cdot 9,34 \cdot 0,34 \cdot 7,36 \cdot 5$, and $37 \cdot 5$ days within a spread of \pm 0.3 days. FraserSmith (1972) reported lines at $26 \cdot 9,27 \cdot 2,27 \cdot 6,30 \cdot 5$ and $37 \cdot 4$ days which tally with our list except that we observed a strong peak at 27.6 days for several parameters but only a smaller peak at $27.2 \pm 0.2$ days for some parameters only. Our 29.5 days matches the synodic period of the moon, which Fraser-Smith seems to have missed.

(4) Analysis of weekly means of Ap for 1965, 1966, 1967, 1968 and 1969 separately showed a slight possibility of a shift of some periodicities from lower to higher values from quiet sun to disturbed sun years.

(5) Analysis of continuous monthly means for several years revealed several significant peaks common to many parameters notably at $48,27,15,12-13,7 \cdot 6,6 \cdot 6,5 \cdot 6,4 \cdot 6,3 \cdot 8,3 \cdot 4$, 3.0,2.5 and $2 \cdot 1$ months. More extended data for Ap (28 years) gave 10.2, 6.8, $5 \cdot 1$ and $4 \cdot 2$ years as additional periodicities and these tally with those obtained by Fraser-Smith. These peaks seem to be harmonics of a 20-22 year double sunspot cycle.

Fraser-Smith (1972) has reported a detailed analysis and comparison of Ap and sunspot number spectra and finds that many lines are common to the two, indicating 
that the changes in Ap activity are due to a direct influence of sunspot activity. He has also referred to the possibility of some frequencies occurring as side lobes of some strong frequencies. From some samples of artificial single sinusoids that we examined, there was no evidence of any side lobes. We presume, therefore, that the Burg and FABNE spectral analysis does not give any significant side lobe effects. If so, the frequencies we observed must have real physical causes. The line near 4.1 years observed by us and Fraser-Smith has no matching line in the sunspot spectrum; but there could be a connection through the coronal 5305 A line which seems to be well correlated to Ap (Gnevyshev 1967).

The MESA anialysis by Currie (1976) of the 100 annual values of the aa magnetic activity index of Mayaud after removing the mean and linear trends showed a 79 year peak (which he correlated with the Gleissberg sunspot cycle of 80 years, Waldmeier, 1966), the fundamental solar magnetic cycle (SMC) at 22 years, the fundamental solar cycle (sc) at 11 years and several other peaks, most of which fitted into harmonic sequences of the SC and SMC. The MESA analysis of the same $a a$ index series by Courtillot et al (1977) showed that the larger periods were considerably affected when trends were removed. Also, the choice of LPEF seemed to play an important role e.g. splitting of the SC (11-year peak) for LPEF exceeding 30 . Whether this peak splitting indicates a genuine physical process (Radoski et al 1975) or is an artifact of the MESA method (Courtillot et al 1977) is a moot question. Our analysis of artificial samples (Kane 1979) showed that closely spaced doublets or triplets could be resolved only with LPEF exceeding $50 \%$ of data length (if at all).

Our present analysis shows many more lines than those reported by the abovementioned authors. Probably, some of our lines are due to spectral instabilities. Some are probably representative of transient powers in particular frequency bands in data of short intervals and hence disappear in longer data sets. However, a possible relationship between the periodicities in solar activity and periodicities in geomagnetic indices seems to be reasonably certain.

\section{Acknowledgements}

Thanks are due to the various people and agencies responsible for producing and publishing the geomagnetic indices and other data. This work was partially supported by FNDCr, Brasil, under contract FINEP-537/CT.

\section{References}

Abdel-Wahab A and Goned A 1974 Planet. Space Sci. 22537

Akaike H 1969 Ann. Inst. Stat. Marh. 21243

Anderson N 1974 Geophysics 3969

Angell J K and Korshover H 1964 J. Atmos. Sci. 21479

Barrodale I and Erickson R E 1980a Geophysics 45420

Barrodale I and Erickson R E 1980b Geophysics 45433

Behannon K W and Ness N F 1966 The design of numerical filters for geomagnetic analysis, NASA Tech. Note TN D.3341, July

Bevington P R 1969 Data reduction and error analysis for the physical sciences (New York: McGraw Hill) pp. $164-176$ 
Blackman R B and Tukey J W 1958 The measurement of power spectra (New York: Dover)

Burch J I 1973 J. Geophys. Res, 781047

Burg J P 1967 Maximum entropy spectral analysis, Paper presented at the 37th meeting, Soc. of Explor.

Geophys., Oklahama City, Oklahama, October

Burg J P 1972 Geophysics 37375

Chen W Y and Stegan G R 1974 J. Geophys. Res. 793019

Cheronsky E J 1966 J. Geophys. Res. 71965

Courtillot V and Le Mouël J L 1976 J. Geophys, Res. 812941

Courtillot V, Le Mouël J L and Mayaud P N 1977 J. Geophys. Res. 822641

Currie R G 1973 Astrophys, Space Sci. 21425

Currie R G 1974 J. Geomagn. Geoelectr. 26319

Currie R G 1976 Astrophys. Space Sci. 39251

Fraser-Smith A G 1972 J. Geophys. Res. 774209

Fraser-Smith A G 1973 J. Geophys. Res. 785825

Gnevyshev M N 1967 Sol. Phys. 1107

Jensen O G and Ulrych T J 1973 Astron. J. 781104

Johnston J 1960 Econometric methods (New York: McGraw Hill) pp. 134-135

Kane R P 1977 J. Geomagn. Geoelectr. 29471

Kane R P 1979 J. Geophys. Res. 84965

Kane R P and Trivedi N B 1982 Geophysics 47 173:

Marple L 1980 IEEE Trans. Acoust., Speech, Signal Process. 28441

Mayaud P N 1968 Indices Kn, Ks et Km 1964-1967, National Centre for Scientific Research, Paris

Mayaud P N 1970a Ann. Geophys. 26109

Mayaud P N 1970b Ann. Geophys. 26313

Radoski H R, Fougere P F and Zawalick EJ 1975 J. Geophys. Res. 80619

Russel C T and McPherron R. L 1973 J. Geophys. Res. 7892

Shapley A H 1947 Trans. Am. Geophys. Union 28715

Sugiura M and Poros D J 1971 Hourly values of equatorial Dst for the years 1957 to 1970, GsFC publication X-645-71-278, July

Svalgaard L and Wilcox J M 1975 Sol. Phys. $\mathbf{4 1} 461$

Ulrych T J and Bishop T N 1975 Rev. Geophys. Space Phys. 13183

Ulrych T J and Clayton R W 1976 Phys. Earth Planet. Inter. 12188

Waldmeier M 1966 Astron. Mitt. Eid. Sternwarte Zurich 2741

Yacob A and Bhargava B N 1968 J. Atmos. Terr. Phys. 301907 